\title{
Neural Code of Motor Planning and Execution during Goal-Directed Movements in Crows
}

\author{
Paul Rinnert and ${ }^{\circledR}$ Andreas Nieder \\ Animal Physiology Unit, Institute of Neurobiology, University of Tübingen, 72076 Tübingen, Germany
}

The planning and execution of head-beak movements are vital components of bird behavior. They require integration of sensory input and internal processes with goal-directed motor output. Despite its relevance, the neurophysiological mechanisms underlying action planning and execution outside of the song system are largely unknown. We recorded single-neuron activity from the associative endbrain area nidopallium caudolaterale (NCL) of two male carrion crows (Corvus corone) trained to plan and execute head-beak movements in a spatial delayed response task. The crows were instructed to plan an impending movement toward one of eight possible targets on the left or right side of a touchscreen. In a fraction of trials, the crows were prompted to plan a movement toward a self-chosen target. NCL neurons signaled the impending motion direction in instructed trials. Tuned neuronal activity during motor planning categorically represented the target side, but also specific target locations. As a marker of intentional movement preparation, neuronal activity reliably predicted both target side and specific target location when the crows were free to select a target. In addition, NCL neurons were tuned to specific target locations during movement execution. A subset of neurons was tuned during both planning and execution period; these neurons experienced a sharpening of spatial tuning with the transition from planning to execution. These results show that the avian NCL not only represents high-level sensory and cognitive task components, but also transforms behaviorally-relevant information into dynamic action plans and motor execution during the volitional perception-action cycle of birds.

Key words: bird; crow; motor execution; motor planning; NCL; single-neuron recordings

Significance Statement

Corvid songbirds have become exciting new models for understanding complex cognitive behavior. As a key neural underpinning, the endbrain area nidopallium caudolaterale (NCL) represents sensory and memory-related task components. How such representations are converted into goal-directed motor output remained unknown. In crows, we report that NCL neurons are involved in the planning and execution of goal-directed movements. NCL neurons prospectively signaled motion directions in instructed trials, but also when the crows were free to choose a target. NCL neurons showed a target-specific sharpening of tuning with the transition from the planning to the execution period. Thus, the avian NCL not only represents high-level sensory and cognitive task components, but also transforms relevant information into action plans and motor execution.

\section{Introduction}

Birds are famous for their motor dexterity in a broad range of behaviors, ranging from flying and hunting to communicative displaying and singing. Some of these behaviors, such as nest building or plumage cleaning, involve sophisticated head-beak coordination. Birds such as crows even use elaborate head-beak movements to build and use tools (Weir et al., 2002; Bird and Emery, 2009). Such coordinated actions require acute senses

\footnotetext{
Received Mar. 31, 2020; revised Jan. 19, 2021; accepted Jan. 28, 2021.

Author contributions: P.R. and A.N. designed research; P.R. performed research; P.R. contributed unpublished reagents/analytic tools; P.R. and A.N. analyzed data; P.R. and A.N. wrote the paper.

This work was supported by the Deutsche Forschungsgemeinschaft Grant NI 618/7-1 (to A.N.).

The authors declare no competing financial interests.

Correspondence should be addressed to Andreas Nieder at andreas.nieder@uni-tuebingen.de.

https://doi.org/10.1523/JNEUROSCI.0739-20.2021

Copyright $\odot 2021$ the authors
}

(Iwaniuk and Wylie, 2020), but most of all delicate motor planning and motor execution capabilities (Davies and Green, 1994; Brecht et al., 2019). With the exception of birdsong research, a research area that resulted in detailed knowledge on the neuroanatomy and neurophysiology of song production (Elemans, 2014; Schmidt and Wild, 2014; Murphy et al., 2017; Kersten et al., 2021), and despite the solid understanding of the neuroanatomy of the avian motor system (Dubbeldam, 2000), the neurophysiological mechanisms of motor plans and actions are rarely explored in birds. This is particularly true for cognitively-controlled and intentional motor plans that in corvid songbirds are comparable to those found in primates (Kabadayi et al., 2016; Miller et al., 2019).

Actions are defined by a goal and a display of motor responses to attain that goal. The goal-directedness of actions requires the integration of sensory and cognitive input with motor output. A precondition for this type of sensorimotor 
integration is a neural structure with connections that link the avian brain's sensory and motor hierarchies. We hypothesized that the nidopallium caudolaterale (NCL) would constitute such a sensorimotor link and encode the planning of head-beak motor acts in carrion crows. The NCL is a high-level associative endbrain region in birds, which, despite its distinct anatomy and evolution independent from mammals (Karten, 2015; Briscoe and Ragsdale, 2018), is often compared with the primate prefrontal cortex (PFC; Divac et al., 1985; Güntürkün, 2005). The NCL operates at the apex of the avian endbrain hierarchy and receives processed information from secondary sensory regions (Kröner and Güntürkün, 1999). Neurons in the NCL are known for their high-level sensory and cognitive coding properties. For instance, NCL neurons represent numerical quantity (Ditz and Nieder, 2015, 2016, 2020), encode sensory features in working memory (Veit et al., 2014; Rinnert et al., 2019), signal cross-temporal and cross-modal associations (Moll and Nieder, 2015; Veit et al., 2015), display value-related activity (Dykes et al., 2018), represent conscious sensory contents (Nieder et al., 2020) and code abstract behavioral principles (Veit and Nieder, 2013).

However, the NCL as a genuine sensorimotor hub is also known to send anatomic projections to dedicated motor structures in the avian brain (Kröner and Güntürkün, 1999) and therefore may also adopt premotor functions. This is reminiscent of the prefrontal to premotor cortex circuitry in primates. The premotor cortex combines sensory-instructional and actionrelated representations to guide voluntary movements (Wise et al., 1997; Hoshi and Tanji, 2000, 2006; Churchland and Shenoy, 2007; Thura and Cisek, 2014; Chandrasekaran et al., 2017). Neurons in the primate premotor cortex are modulated by sensory signals that cue motor responses to represent forthcoming action choices and represent abstract decision-related concepts before action choices are fully specified (Cisek and Kalaska, 2005; Nakayama et al., 2008). Even neurons in the basal gangliacortical circuits are modulated by behavioral context such as cued versus self-timed movement initiation (Gdowski et al., 2001; Lee and Assad, 2003). We therefore hypothesized that the crow NCL may be an ideal candidate structure to transform sensory input into goal-directed motor commands. In the current study, we explored the neuronal correlates of the planning and execution of goal-directed head-beak reaching movements in carrion crows.

\section{Materials and Methods}

Animals

We used two male carrion crow (Corvus corone; one and two years old, respectively) from the institutes facility. Both crows were hand-raised and housed in social groups in indoor aviaries (Hoffmann et al., 2011). The crows were on a controlled feeding protocol during training and recording day. Body weight was measured daily. Food was given as reward during the experiment, or if necessary after. Water was provided ad libitum in the aviaries and during the experiments. All procedures were conducted according to the guidelines for animal experimentation and approved by the responsible national authorities, the Regierungspräsidium Tübingen, Germany.

\section{Surgery and recordings}

Surgery was performed under general anesthesia with a mixture of ketamine $(50 \mathrm{mg} / \mathrm{kg})$ and xylazine $(5 \mathrm{mg} / \mathrm{kg})$. The crows were placed in a stereotaxic holder and craniotomy was performed to target the medial part of NCL (Sen et al., 2019) with coordinates $5 \mathrm{~mm}$ anterior-posterior and $13 \mathrm{~mm}$ medio-lateral in the left hemisphere. Two custom-build micro drives with four electrodes each ( $2 \mathrm{M} \Omega$, Alpha Omega Co) were implanted at the craniotomy. In addition, a connector for the headstage and small holder for the reflector were implanted. Before each recording session while the crow was perched inside the chamber and waited for the start of the trials, electrodes were gradually advanced until good neuronal signal was detected on at least one channel. Neurons were not preselected for involvement in the task. Single neuron separation was done offline (Plexon Offline Sorter, version 2.6.2). For analysis of neuronal activity, we only considered neurons with at least $1-\mathrm{Hz}$ average firing rate (to avoid statistical problems of $0-\mathrm{Hz}$ entries during firing rate analysis), that were recorded for at least 20 trials for each side cue $(n=408)$.

\section{Experimental setup}

Experiments were conducted in a darkened operant conditioning chamber. The bird was perched in front of a touch screen (ART development MT1599-BS), that was used for stimulus presentation and to record behavioral responses. Food reward was delivered by a custom-built automated feeder below the touch screen. Food reward consisted of food pellets (Beo special, Vitakraft) and mealworms (Tenebrio molitor larvae). Additional feedback was provided by a lamp that illumined the feeder opening whenever a reward was delivered after a correct trial. In addition, a loudspeaker (Lasmex S-03) located behind the touch screen played one sound for correct, and another sound for incorrect responses; these sounds thus provided positive and negative acoustic feedback, respectively. An infrared light barrier in front of the screen detected, when the bird was positioning its head in front of the screen. A CORTEX system (National Institute of Mental Health) was used to carry out the experiments and collect behavioral data. Neuronal data were recorded using a PLEXON system (Plexon Inc.).

\section{Behavioral protocol}

The birds were trained on a spatial delayed response protocol (Fig. 1A). A go-stimulus (white square, $3.3 \times 3.3^{\circ}$ visual angle) instructed the bird to move its head into the light barrier to start a trial. If the head was moved out of the light barrier before beginning of the response period, the trial was aborted. Each trial started with a 300 -ms target cueing period, in which two gray frames $\left(4.8 \times 4.8^{\circ}\right.$ visual angle $)$ introduced two possible target locations on the touchscreen. There were eight possible target locations, that were circularly arranged around the center of the screen (Fig. 1B). In each trial, the two possible target locations had the same vertical position, so that there was always one possible target location on the left and one on the right side of the screen. In the side planning period, a side cue appeared in the center of the screen, in addition to the possible target locations. The side cue instructed the bird to later perform a movement to either the left or right target location (Fig. 1C). The side cues for left and right differed in either their color (color cues, $0.2 \times 0.2^{\circ}$ visual angle), with a red square instructing the left side and blue square instructing the right side, or their shape (shape cues, $0.2 \times 0.6^{\circ}$ and $0.6 \times 0.2^{\circ}$ visual angle), with a horizontal bar instructing the left side and a vertical bar instructing the right side. These two different sets of side cues allowed us to disentangle the neuronal coding of sensory features of the side cues (colors and shapes) from the instructions themselves (left vs right). The onset of the response period was indicated by a vanishing of the central side cue. In order to force the crows to pay attention to the central side cue and to continuously maintain fixation during the planning period, the length of the planning period was randomly varied between 1 and $3 \mathrm{~s}$. In addition, the crows were forced to respond as rapidly as possible and within $800 \mathrm{~ms}$ by pecking to the instructed target location in the subsequent response period. Correct and swift responses were rewarded with food and a "correct" sound, whereas responses after $800 \mathrm{~ms}$ or to the wrong target location resulted in abort of the trial with an "error" sound and without food reward. Importantly, the side cue and the target location information were continuously present during the planning period, so that there was no need to memorize the target location. At the same time, the variable length of the planning period and the short response window forced the crows to prepare the correct response beforehand.

In order to explore whether neuronal activity during the planning period was predictive of the crows' responses, neutral cues were introduced in twenty percent of the trials (self-chosen target trials; Fig. 1A, bottom trial sequence). These ambiguous or neutral cues were 
A

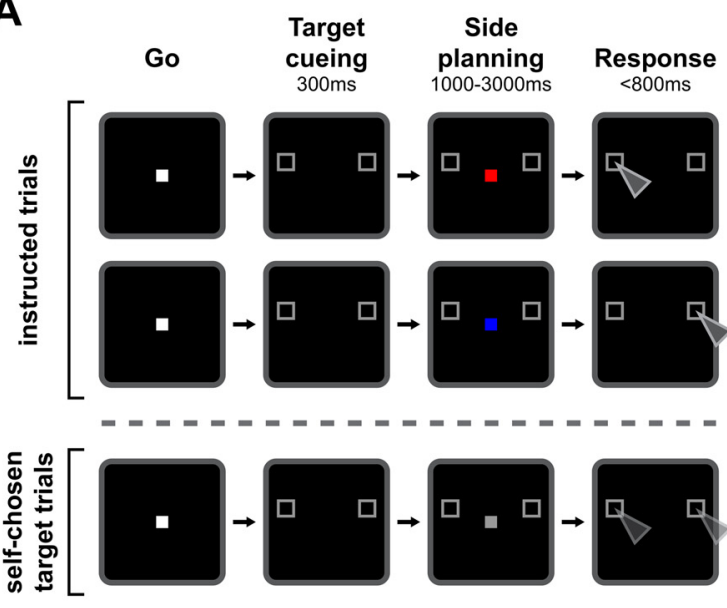

B Target locations

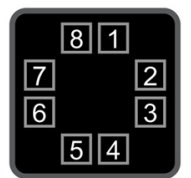

C

\section{Side Cues}

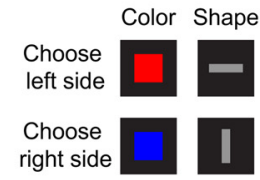

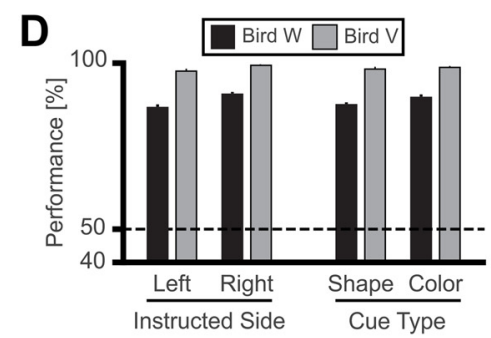

Figure 1. Spatial delayed-response task and behavioral performance. $A$, Behavioral task. In the Go period, the crow moved its head into a light barrier centrally in front of the screen to start a trial. In the following cue period, two out of eight gray frames indicated the possible target locations (target cueing period). The presented possible target locations were horizontally aligned. The target cueing period was followed by the side planning period. Here, a side cue was additionally presented in the center of the screen. The side cue instructed either the left or right target location for the later response. Side cues for left and right differed in either their shape or color. The side planning period length was variable between 1 and $3 \mathrm{~s}$. During the side planning period, both possible target locations and the side cue were presented continuously, making a memorization unnecessary. Once the central side cue vanished, the bird had to rapidly perform a movement to the instructed location within $800 \mathrm{~ms}$. Variable planning period length and short response window made a planning of movement beforehand necessary. In instructed trials, the side cue instructed either the left or right target location. In self-chosen target trials, a neutral cue instructed neither location and either choice was randomly rewarded. $\boldsymbol{B}$, All possible eight target locations. C, Color and shape side cues, instructing left and right target location. $\boldsymbol{D}$, Average behavioral performance for left and right side instructed and different cue types for bird W (black bars) and bird V (grey bars). Error bars indicate SEM.

characterized by a sensory appearance that was in between the instructive side cues (such as gray squares instead of red and blue squares) and were therefore intentionally ambiguous in their instructive meaning. For such ambiguous cues, choices to either target location were rewarded randomly in $50 \%$ of the trials to prevent learning. Because of individual preferences in both birds, choices within a session were biased to a specific side. We therefore adapted the appearance of the neutral cue over time (by rendering it more similar to one of the learned side cues) to obtain balanced location choices.

For analysis of behavior, all error bars and error measures indicate SEM, that is the SD divided by the square root of the sample size. To test performance against chance level we performed a binomial test with 0.5 probability (i.e., $50 \%$ chance performance) on the average recording day performance.

\section{Neuronal selectivity analyses}

To analyze a neuron's premotor selectivity during the planning period, we used the average spike rate during a 900 -ms window, starting $100 \mathrm{~ms}$ after beginning of the planning period to account for response latencies in NCL neurons (Veit et al., 2014). Integration over a relatively long 900-ms window was chosen to calculate reliable directional selectivity and robust directional vectors, but also to have NCL data comparable to monkey PFC results (Takeda and Funahashi, 2002). During the planning period, the crow was instructed by one of four side cues ("side cue types"; Fig. 1C) to prepare a movement to either the left or right side of the screen. In addition to target side (left-right), the crow was additionally cued (by the targets) to prepare a movement to one of the eight target locations (target locations 1-8; Fig. 1B), each target location was either on the left or right side of the screen (target locations 1-4 on the right side, target locations 5-8 on the left side).

In order to analyze neuronal responses for selectivity for both side and location (a subgroup within side) with one analysis, we chose a nested ANOVA (or hierarchical ANOVA). The nested ANOVA is designed to be able analyze factors when these form subgroups within groups. In our design the factor location (as a subgroup) is nested within the factor side, meaning that each location is only found on one side (McDonald, 2014). Using the nested ANOVA allowed us to analyze selectivity to the three main factors "side cue," "target side," and "target location," of which the main factor target location was embedded in the main factor target side. To account for the hierarchical relationship of target location within target side, we calculated a three-factorial nested ANOVA [factors target side, target location (nested within side), and side cue type]. A neuron was defined as "planning-selective" if it showed a significant ANOVA effect for the factors target side and/or target location $(p<0.05)$ and did not show an effect for the factor side cue type $(p>0.05)$. Selective neurons with only a selective factor target side were termed "side-selective," while neurons with only a selective factor target location were termed "location-selective." Neurons with both a selective factor target side and location were termed "side-and-location selective."

To analyze selective activity during movement execution in the response period, we analyzed the average spike rate during a $350-\mathrm{ms}$ window ending $50 \mathrm{~ms}$ before a peck on the screen. During movement execution, the crow performed an action to one of eight target locations (factor target location) on the left or right side of the screen (factor target side) in response to the rule cues (factor side cue type). In addition, a fourth factor "planning period length" was introduced to account for the variable lengths of the planning period (between 1 and $3 \mathrm{~s}$ ). Based on these four factors, and to account for the hierarchical relationship of target side and location, we calculated a four-factorial nested ANOVA [factors target side, target location (nested within side), side cue type, and planning period length]. A neuron was defined as "execution-selective," if it showed a significant effect for the factors target side and/or target location $(p<0.05)$ and did not show an effect for the factor side cue type of planning period length $(p>0.05)$. In the same way as for the planning-selectivity analysis, neurons with only a selective factor target side were termed side-selective, neurons with only a selective factor target location were termed locationselective, and neurons with both significant factors for target side and location were termed side-and-location selective.

We analyzed whether the identification of selective neurons was influenced by the different numbers of trials for each neuron. In the total population of reported neurons with sufficient trial numbers $(n=408$ neurons), the number of trials recorded ranged from 82 to 565 trials per neuron (with an average of 272 trials per neuron). We split the neuron population into a subpopulation with "high number of trials" (recorded for $>272$ trials; $n=194$ ) and a subpopulation with a "low number of trials" (recorded for $<272$ trials; $n=214$ ). In the planning period, we found comparable numbers of 60 selective neurons in high number of trials group versus 57 selective neurons in low number of trials group $\left(\chi^{2}\right.$, 
A
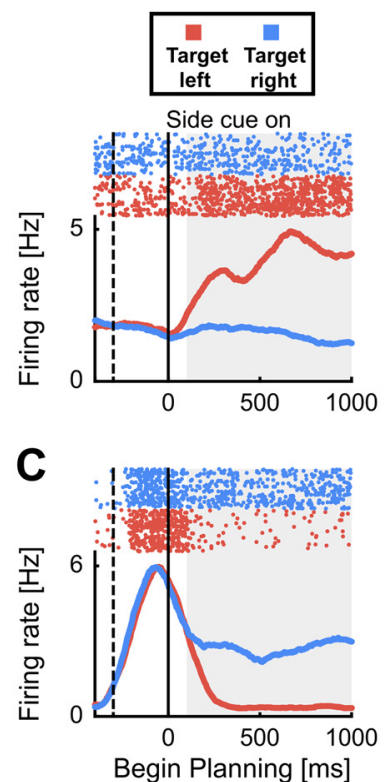

Side cue modality

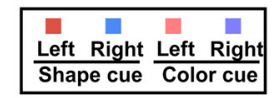

Side cue on
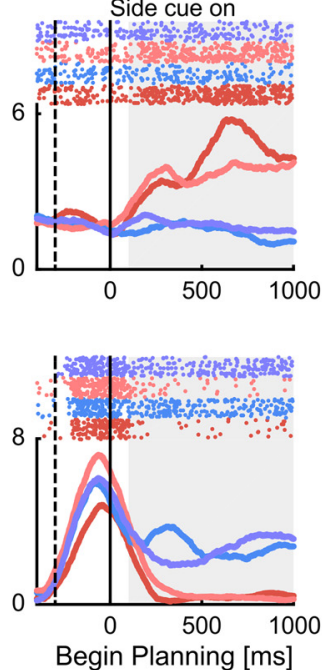

Target location

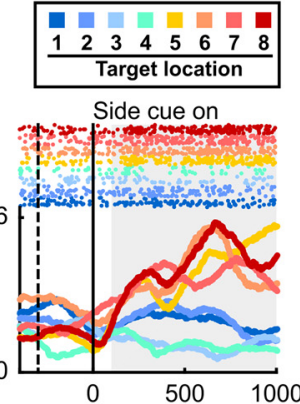

B

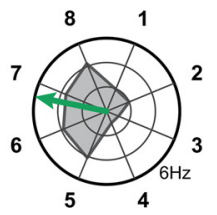

D

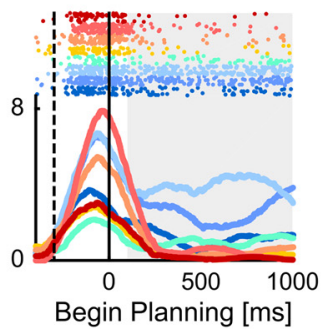

D

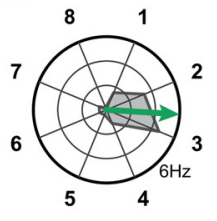

Figure 2. Neuronal responses to target locations during planning period. Example neurons with selective activity for target side and location. $A, C$, The first three panels show a dot-raster histograms (top) with corresponding spike-density functions (bottom) aligned to begin of the planning period. In dot-raster histograms, each dot represents an action potential, and each row represents a trial. Spike-density functions show the average firing rate smoothed with a 300-ms Gaussian kernel. Solid vertical lines represent begin of the planning period and dashed vertical lines the begin of the target cueing period. The left panel shows trials sorted by the instructed side. The middle panel shows trials sorted according to all individual side cue types. The right panel shows trials sorted for all eight different instructed target locations. $\boldsymbol{B}, \mathbf{D}$, Polar plots show the firing rate for all eight target positions averaged over the gray shaded area in $\boldsymbol{A}, \boldsymbol{C}$. The arrow represents the preferred direction of the neuron. $\boldsymbol{A}, \boldsymbol{B}$, Side-selective example neuron showing preference equal for all target locations on the left side. $\boldsymbol{C}, \boldsymbol{D}$, Side-and-location selective example neuron showing preference for target positions on the right side, and additional preference for target locations 2 and 3 within the right side.

$p>0.05)$. In the execution period, the frequency of 92 selective neurons in the high number of trials group was slightly higher compared with the 66 selective neurons in the low number of trials group $\left(\chi^{2}, p<0.05\right)$. Thus, identification of selective neurons was not affected by trial sample size during the planning period, but during the execution period.

\section{Calculating the preferred direction and side for single neurons}

To calculate the preferred direction of a neuron, we calculated the average firing rate during correct trials for each target location individually. The average firing rate for an individual target location was used to create a vector with the length of the average firing rate (in Hertz) and the direction of the target location (in degrees). These eight target location vectors represent the average response strength of the neuron in trials with the respective target location. We defined the preferred direction (in degrees) as the direction of the vector resulting from vector addition of all eight target location vectors. Examples of the preferred directions are displayed as arrows in Figure 2B,D. Hence, in this calculation of preferred direction, each target location obtained an equal vote, which resulted in a more fine grained direction vector that factored in all target location vectors in contrast to a classical "winner takes all" calculation. In the classical winner takes all approach, the preferred direction would be defined as the direction of the target location vector with the highest firing rate. Both vector addition and winner-takes-all measures yielded similar results: we compared both measures by calculating a circular-circular-correlations for all planning-selective and execution-selective neurons (Berens, 2009) and found a significant correlation between both results $\left(p<0.05, \rho_{\mathrm{cc}}=0.40\right.$; angular difference: mean $=6.9^{\circ}$, standard deviation $\left.(\mathrm{std})=74.8^{\circ}\right)$. The preferred location of a neuron was defined as the target location closest to the preferred direction of the neuron. The preferred side of a neuron was defined as the preferred direction vector discretized into left and right.

Area under the receiver-operating-characteristic curve (AUROC) comparison of side-selective neurons in instructed and self-chosen target trials To compare the neuronal activity of side-selective neurons during instructed trials versus self-chosen trials, we compared the AUROC values for individual neurons during both conditions. The AUROC (area under the receiver operator characteristic) is a measure derived from signal detection theory (Green and Swets, 1966). The AUROC quantifies the overlap of two (firing rate) distributions for a specific neuron recorded during two different conditions. The AUROC value provides information about how well a neuron based on its firing rates can differentiate between two states/conditions. The AUROC can take on values from 0 to 1 . A value of 0.5 indicates that the two firing rate distributions are completely overlapping and therefore the neuron is not differentiating between the two conditions. A value of 1 or 0 indicates complete segregation of the two firing rate distributions and thus optimal discriminability of two conditions based on the neurons' responses. In a first step, we calculated an AUROC value of preferred versus nonpreferred side during instructed trials for all side-selective planning-neurons $(n=84)$. The preferred side was defined by the preferred direction categorized into left and right (as described above). Because we defined the preferred side as the reference distribution for each side-selective neuron, all AUROC values were above 0.5 by definition. Average firing rates of a $1000-\mathrm{ms}$ window starting $100 \mathrm{~ms}$ after begin of the planning period were analyzed. Next, we calculated the AUROC value during selfchosen target trials for all side-selective neurons that were recorded for at least five self-chosen trials for each side (35/84). For this analysis we defined the reference distribution based on the preference in instructed trials, so that values above 0.5 represent preference (i.e., higher firing rate) for the same side during instructed and self-chosen target trials. We performed a Wilcoxon signed-rank test to determine whether the AUROC values during self-chosen trials were significantly above 0.5 in our population of selective neurons.

\section{AUROC analysis for error trials}

We performed an AUROC analysis including both correct and error trials for all side-selective neurons during the planning period $(n=84)$, to investigate whether the encoding of the later target side was also present in error trials. Similar as done in Russ et al. (2008), we first calculated the AUROC value for each side-selective neuron over both correct and error 
trials combined (trial types sorted by the side of the later response, e.g., correct trials with left side instructed and error trials with right side instructed were grouped into the same category). As in the previous AUROC analysis of self-chosen target trials, we calculated AUROC values of preferred versus non-preferred side and defined the preferred side during correct trials as reference distribution. This results per definition in AUROC values above 0.5 for all side-selective neurons, when using only correct trials. In a first step, we compared whether the AUROC values were also significantly above 0.5 when including both correct and error trials sorted by the later target side using a one-sided $t$ test. In a second step, we calculated the AUROC values only using error trials sorted by the later target side for all side-selective planning neurons that were recorded for at least five error trials for each side $(n=17)$ and tested whether they were significantly above 0.5 (one-sided $t$ test).

\section{Support vector machine (SVM) prediction of target in self-chosen target trials}

To assess whether the neuronal activity during planning in self-chosen target trials was predictive of the later chosen target, we used SVM classifier that we trained on instructed trials to predict the target in self-chosen target trials. This analysis was performed once for the prediction of target side and separately for the prediction of individual target locations. For prediction of target side, all neurons regardless of selectivity with at least five self-choices to each side and 40 instructed trials for each side entered the analysis $(n=248)$. For prediction of target location, all neurons regardless of selectivity with at least two self-choices to each target location and 10 instructed trials for each location entered the analysis $(n=104)$. Average firing rates of a $1000-\mathrm{ms}$ window starting $100 \mathrm{~ms}$ after begin of the planning period were analyzed. Each classifier was trained on $m$ randomly chosen instructed trials of each neuron for each side/ location ( $m=40$ for target side and $m=10$ for target location). During training of an SVM classifier, the classifier creates a population of pseudo-simultaneous recorded neurons and tries to create boundary vectors separating the to be classified groups with the largest margin. For prediction of target side or location in self-chosen target trials, we randomly drew a self-chosen target trial of each neuron for each group to create pseudo-population activity during self-chosen target trials. This process was repeated 100 times resulting in 100 self-chosen test trials for each side/location. The prediction performance was defined as average of correct predictions over the 100 self-chosen target trials. The process of training the classifier and prediction of test trials was repeated 100 times to account in differences in trial selection. To compare the results to chance level, we repeated the same analysis but trained the classifier with permuted trial labels resulting in a prediction performance under conditions without neuronal selectivity. Prediction performance was defined to be above chance level if the average was above the 95th percentile of performances derived with the permuted classifier. We used the LIBSVM library (version 3.23; Chang and Lin, 2011) with all parameters set to default for training of the classifier. The default kernel for creation of boundaries is a radial basis function, but we also obtained qualitatively similar results using a linear kernel.

\section{SVM confusion matrix analysis}

To investigate, how well the neuronal activity during instructed trials predicts individual target locations, we performed a cross-validation analysis using SVM classifier. In this analysis a SVM classifier was repeatedly trained on instructed trials to predict the target location of other instructed trials. Planning and execution period were analyzed separately. For the planning period, we analyzed a 1000 -ms window starting $100 \mathrm{~ms}$ after beginning of the planning period, to account for visual latency. For the execution period, we analyzed neuronal activity in a 350 $\mathrm{ms}$ window ending $50 \mathrm{~ms}$ before the peck, accounting for the time of movement execution. All neurons, that were recorded for at least 30 trials for each target location entered this analysis $(n=175$; same population for planning and execution period). We performed a 5 -fold crossvalidation analysis on a classifier fed with 30 randomly chosen instructed trials of each target location for each neuron, resulting in 240 or $30 \times 8$ data points. In a 5 -fold cross-validation, the dataset is split up into five equal sized parts of data points. In the next step the classifier is trained on four parts $(24 \times 8$ trials of the total of $30 \times 8$ trials $)$ and tested on the remaining part $(6 \times 8$ trials). This process is repeated until all parts were predicted once. In this way cross-validation prevents an overestimation of classification performance, by ensuring that the data used for testing of the classifier is not used during its training. A confusion matrix was calculated by comparing the true label of a data point with the predicted label during cross-validation. The whole process was repeated 100 times to account for differences in trial selection. The average decoding performance was defined as the average percent correct predictions of the classifier over the repetitions. To compare the decoding performance to chance level, we calculated the average percent of correct predictions using shuffled prediction labels. We defined decoding performance to be significantly above chance level if the average decoding performance was above the 95th percentile of performances with shuffled labels. We used the LIBSVM library (version 3.23; Chang and Lin, 2011) with all parameters set to default for training of the classifier. The default kernel for creation of boundaries is a radial basis function, but we also obtained qualitatively similar results using a linear kernel.

\section{Position selective population activity relative to preferred direction}

To investigate the time course of population activity of selective neurons, we calculated the average response of all location-selective neurons relative to their preferred location in a sliding window. For this analysis, we calculated the preferred location of all neurons that were location-selective or side-and-location selective either during the planning and/or execution period ( $n=142$; not including side-selective neurons). The preferred location of a neuron was defined as the target location that was closest to the calculated preferred direction during motor planning. The baseline activity of a neuron was defined as the average firing rate across all correct trials in the 500-ms window before the target cueing period. For each neuron, we calculated the average firing rate in correct trials in which the instructed location coincided with the preferred location. We then calculated the average firing rate over trials in which the instructed location was one to four locations clockwise and counterclockwise next to the preferred location. Note that the location four steps clockwise from the preferred location is the same as the location four steps counterclockwise (8 locations in total). This average firing rate for preferred and neighboring locations was calculated in a sliding window of 200-ms width (value taken at center of window) and in steps of $1 \mathrm{~ms}$. We subtracted the average baseline activity to compare the activity at each time point to the baseline activity of the neurons. Finally, this firing rate difference was averaged at each time point across all 142 location-selective neurons to generate a population response matrix. Neuronal activity during motor planning was analyzed in a $1400-\mathrm{ms}$ window starting $400 \mathrm{~ms}$ before the rule cue onset (begin side planning period). This window accounts for the target cueing period and the first second of the side planning period. Neuronal activity during the motor execution period (Fig. 7B), was analyzed in an 800-ms window starting $500 \mathrm{~ms}$ before the peck on the screen was registered. This window accounts for the time of movement execution and reaches into the reward period. Both analysis matrixes of the planning (Fig. $7 A$ ) and execution period (Fig. $7 B$ ) were aligned according to location preference in the planning phase.

\section{Results}

\section{Behavior}

Both birds performed the spatial delayed response task (Fig. 1A) above chance level for all recording days $(n=125)$. The average performance across all eight target locations was above $85 \%$ for both crows (crow W: $88.7 \pm 4.6 \%$; crow V: $98.6 \pm 2.3 \%$ ). The same high performance was observed for left-side and right-side targets separately (left side: $86.8 \pm 5.9 \%$ and $97.8 \pm 4.0 \%$; right side: $90.8 \pm 3.9 \%$ and $99.6 \pm 0.8 \%$ for crow $\mathrm{W}$ and crow $\mathrm{V}$, respectively; binomial test against equal distribution $p<0.05$; Fig. 1D). Performance for each type of side cue was similarly high (color cues: $89.9 \pm 5.5 \%$ and $98.9 \pm 1.8 \%$; shape cues: $87.7 \pm 4.1 \%$ and $98.4 \pm 3.8 \%$ for crow $\mathrm{W}$ and crow $\mathrm{V}$, respectively; binomial tests against equal distribution $p<0.05$; Fig. 
A

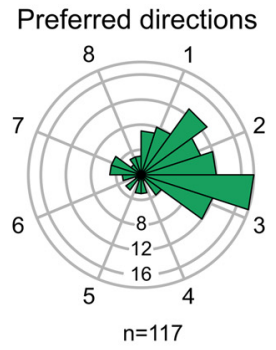

B

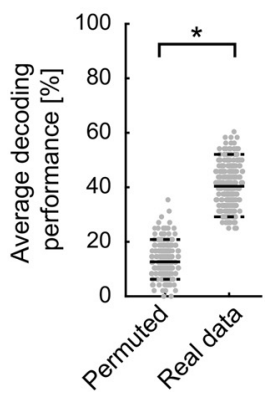

To quantify the involvement of NCL neurons in encoding of target side or location, we used analyses of variance [threefactorial nested ANOVA with factors target side (left or right), target location (eight locations), and side cue type (color or shape)]. Average firing rates per neuron were analyzed in a $900-\mathrm{ms}$ interval starting $100 \mathrm{~ms}$ after side cue onset (Fig. $2 A, C$ ). We selected those $28.7 \%$ (117/408) exclusively "planning-selective neurons" neurons that were selective either to the target side, the target location, or both $(p<0.05$ for factors side and/or location), but showed no selectivity to the type of the side cue ( $p>0.05$ for factor side cue type). Most (61/117) of these planning-selective neurons were only modulated by the target side and not individual target locations (similar to the neuron in Fig. 2A,B). Neurons abstractly encoding target side

$1 D)$. Both crows responded quickly; it took bird $\mathrm{W}$ on average $516 \mathrm{~ms}$ and bird V $507 \mathrm{~ms}$ to respond to the instructed location.

In self-chosen target trials with a neutral cue, both birds showed a side bias. In order to attenuate side bias, the neutral cue was adapted according to the previous day bias (by adjusting color and/or shape). Overall, the right-side target was chosen more often $(76.4 \pm 19.6 \%$ for crow $W$ and $57.1 \pm 37.7 \%$ for crow V). Irrespective of this bias, both sides were chosen sufficiently often to permit an analysis of neuronal firing patterns during self-chosen target trials.

\section{Neuronal data}

Single neurons in NCL encode target side and/or location during planning

We recorded the activity of 408 single neurons in the left NCL while the two crows performed the task (239 neurons in crow W, 169 neurons in crow V). The activity of neurons was modulated either categorically as a function of target side (left vs right), or more selective as a function of individual target locations.

The example neuron in Figure $2 A$ categorically increased its activity in trials in which a target location was on the left side, the preferred side of the neuron (Fig. $2 A$, left panel). The categorical increase of activity to left-side targets was present regardless of the side cue type (color vs bar orientation) that instructed the target side (Fig. 2A, center panel). Moreover, this neuron did not discriminate between individual target locations within the left or right side (Fig. $2 A$, right panel). The neuron's firing rates are virtually identical for all positions on the left side and systematically higher compared with the right side when neuronal activity is averaged over the shortest planning interval (the first second after side cue onset; Fig. 2B, polar plot). This neuron was therefore encoding the categorical side of the planned response.

Figure $2 C$ shows a different example neuron. Its activity was higher during the planning period for right-side targets (Fig. $2 C$, left panel). In addition, this neuron was not differentiating between the type of side cue that instruct a side (Fig. $2 C$, center panel), indicating that it is not encoding the visual features of the side cue. In contrast to the first example neuron (Fig. 2A,B); however, this neuron shows differential activity for individual target locations (Fig. 2D, locations 2 and 3) on the preferred, right side. This neuron therefore differentiated not only between left and right sides, but in addition had a preference for specific target locations. will be called side-selective neurons in the following. The rest of the neurons were either modulated only by individual target locations (33/117) or a combination of target side and individual target location (23/117; see example neuron in Fig. 2C). Neurons with only individual target location selectivity will be called location-selective neurons, while neurons with selectivity for both target location and side will be called "side-and-location selective neurons." Neurons with a selective factor side cue type (40/408 with $p<0.05$ for factor side cue type) were not considered abstract planning neurons as they were affected by the visual features of the side cues. 251 of the 408 recorded neurons were not selective for any analyzed factor during the planning period.

Neuronal motor planning showed strong hemispheric lateralization. We calculated the directional vector indicating the average preferred direction for each planning-selective neuron ( $n=117$; Fig. 2B,D, arrows). Most of the neurons preferred future target locations on the right side (88/117 or $75 \%$ preferring target locations on the right side; binomial test against equal distribution $p<0.05$; Fig. 3A). Because we recorded from the left NCLs in the two crows, neurons signaled planned movements contralateral to the recorded hemisphere.

To evaluate whether the population of all recorded NCL neurons contained robust information that would allow an ideal observer to predict the prospective target side and location, we trained a SVM classifier to predict the target location. The classifier was trained on the firing rates of a fraction of the trials during the planning period, and then tested with the firing rates contained in the other fraction of the trials not used for training (5-fold cross-validation). The classifier was able to predict the instructed target location significantly above chance: the average decoding accuracy was around $40 \%$ and therefore higher than the 95th percentile of decoding accuracies obtained with shuffled data (Fig. 3B, left panel; 95th accuracy percentile of shuffled data were around 20\%).

In a next step, we investigated the classifications of the SVM classifier in more detail by using a confusion matrix (Fig. 3B, right panel). In the confusion matrix, the predictions of the classifier are averaged depending on the true labels, resulting in an $8 \times 8$ matrix for the eight possible target locations. We observed two main effects. First, the classifier had a tendency to misclassify the four targets on either side. As witnessed by the increased classification performance in the upper-left and lower-right quadrants of the 
confusion matrix (Fig. 3B, right panel), target locations within the right side (locations 1-4) and target locations within the left side (locations 5-8) were more often confused than those across both sides. This is in agreement with the previous finding that most of the neurons encoded the instructed prospective left or right side; it confirms the categorical motor plan reflected in the neuronal discharges. Second, the classifier performance also resulted in a diagonal of increased accuracy values where the predicted labels matched the eight true location labels; this result is consistent with the presence of location-selective neurons. The diagonal was stronger for the first four target locations compared with the last four (Fig. $3 B$, right panel). This is equivalent to a lower decoding performance for target locations on the left side (target locations 5-8) compared with target locations on the right side (target locations 1-4). The higher decoding performance for targets on the right side is in agreement with the larger number of selective neurons for that side during the planning period (compare Fig. $3 A$ ). Overall, these findings indicate that neuronal population activity during motor planning represented both the target side and specific target locations within a side.

\section{Neuronal planning activity predicts self-chosen impending targets}

To find out whether the firing of neurons during the planning period had any predictive value for the crows' subsequent side choice, we explored the relationship of neuronal activity with behavior during trials with a neutral cue (self-chosen target trials). Since the neutral cue was not associated with a target side, the crows needed to plan a movement to a self-chosen target. We hypothesized that planning-related neurons would predict the self-chosen target side by showing premotor firing rates that would concur with choices instructed by the two sets of learnt left versus right side cues.

Inspection of single neuron activity suggested that planningselective neurons indeed predicted the upcoming target side. The side-selective example neuron in Figure $4 \mathrm{~A}$ showed an increased firing rate for instructed trials in which the right side was cued. The same neuronal left-versus-right selectivity was seen in selfchosen target trials: firing rates were high when the crow chose the right side, but low whenever it chose the left side in the presence of a neutral cue. The corresponding polar plot in Figure $4 B$ shows that the neuronal activity to each of the eight locations was very similar for trials with instructed and self-chosen targets. This indicated that neuronal selectivity under uncertainty was predictive of the later target side.

To quantify the side predictive power of all selective neurons, we applied signal detection theory (Green and Swets, 1966) and measured the AUROC. The AUROC value quantifies the difference between two distributions of firing rates, in our case the firing rate distributions that occurred while the crows planned to subsequently chose the left-side or right-side target. In other words, the AUROC is a measure of neuronal discriminability. AUROC values of 0.5 signal total overlap of the distributions and therefore no discriminability; values higher than 0.5 up to 1 signal increasing discriminability. These AUROC values were derived and compared for instructed and self-chosen target trials. Per definition, AUROC values of neurons with side-selectivity were higher than 0.5 for instructed trials (mean of $0.62, n=84$; Fig. $4 C$ ). For self-chosen target trials, values higher than 0.5 indicate a preference for the same side as in instructed trials, whereas values lower than 0.5 indicate a change in preference. The mean AUROC for self-chosen target trials (mean of $0.55, n=35$ ) was significantly higher than 0.5 (Wilcoxon signed-rank test, $p<0.05$ ). This
A B
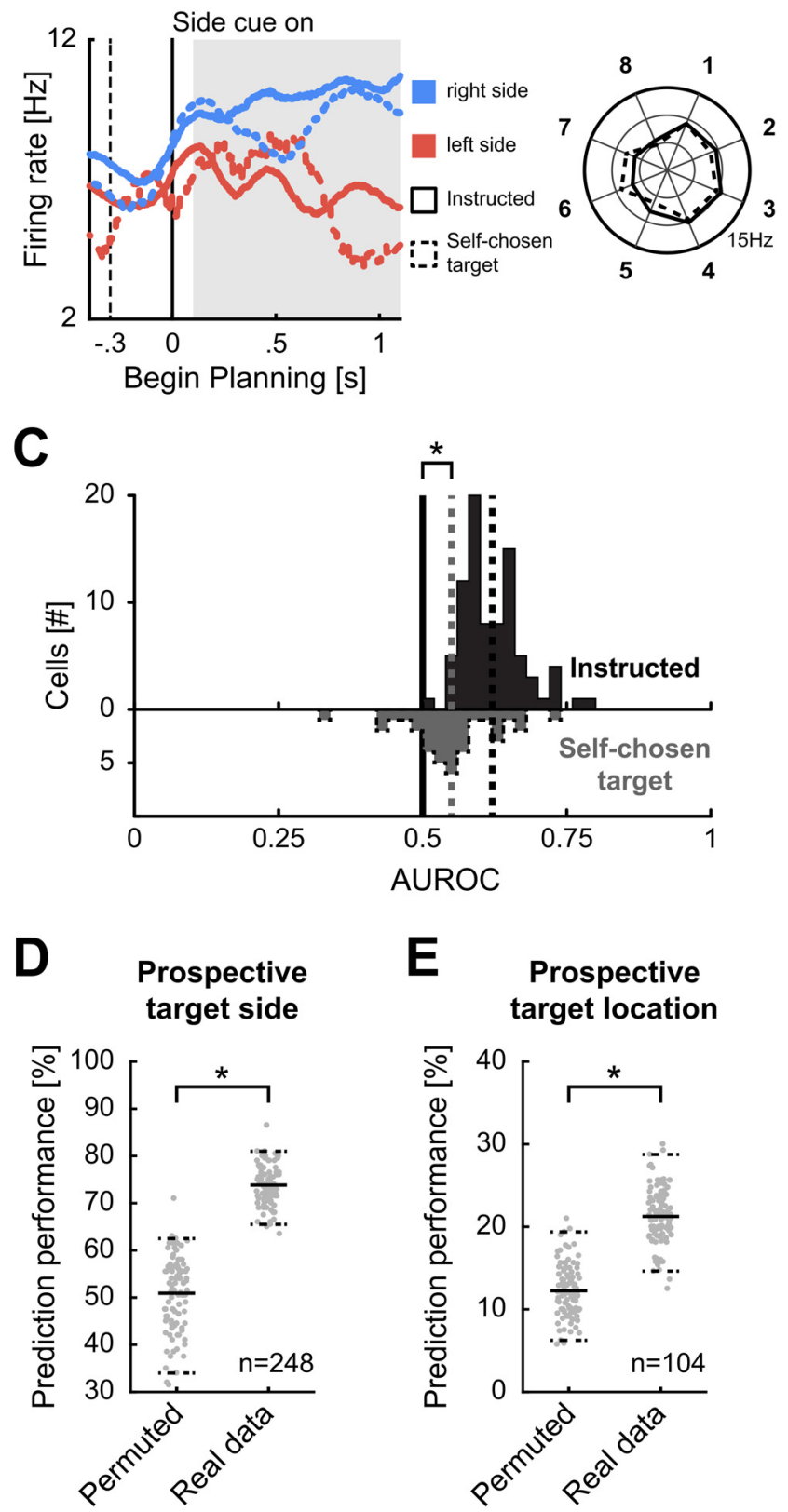

Figure 4. Neuronal responses during self-chosen target trials. A, Spike-density function of an example neuron during instructed and self-chosen target trials. Spike-density function represent the average firing rates smoothed with a 300-ms Gaussian kernel. Solid lines represent neuronal activity during instructed trials, and dashed lines activity during self-chosen target trials. Blue color indicates later movement to the right, and red colors later movement to the left side target location. The solid vertical line represents begin of the side planning period, and the dashed vertical line the begin of the target cueing period. During instructed trials, the neuron has a higher firing rate for trials with target locations on the right side. In self-chosen target trials, the firing rate resembles the firing rate of instructed trials with the same target side. $\boldsymbol{B}$, Polar plot shows the average firing rates for all eight target positions averaged over the gray shaded area in $\boldsymbol{A}$. Average firing rates of instructed and self-chosen trials with the same later target location are similar. $C$, AUROC values for side-selective neurons for instructed and self-chosen trials during planning. $\boldsymbol{D}$, Performance of SVM classifiers trained on instructed trials to predict prospective target side in self-chosen target trials. Right distribution is the prediction performances of classifiers trained on real data, left distribution the prediction performance of classifiers trained on data with permuted labels. Horizontal solid line represents the average prediction performance, dashed lines represent the fifth and 95th percentile of predictions. $\boldsymbol{E}$, Performance of SVM classifiers trained on instructed trials to predict prospective individual target locations in self-chosen target trials. 

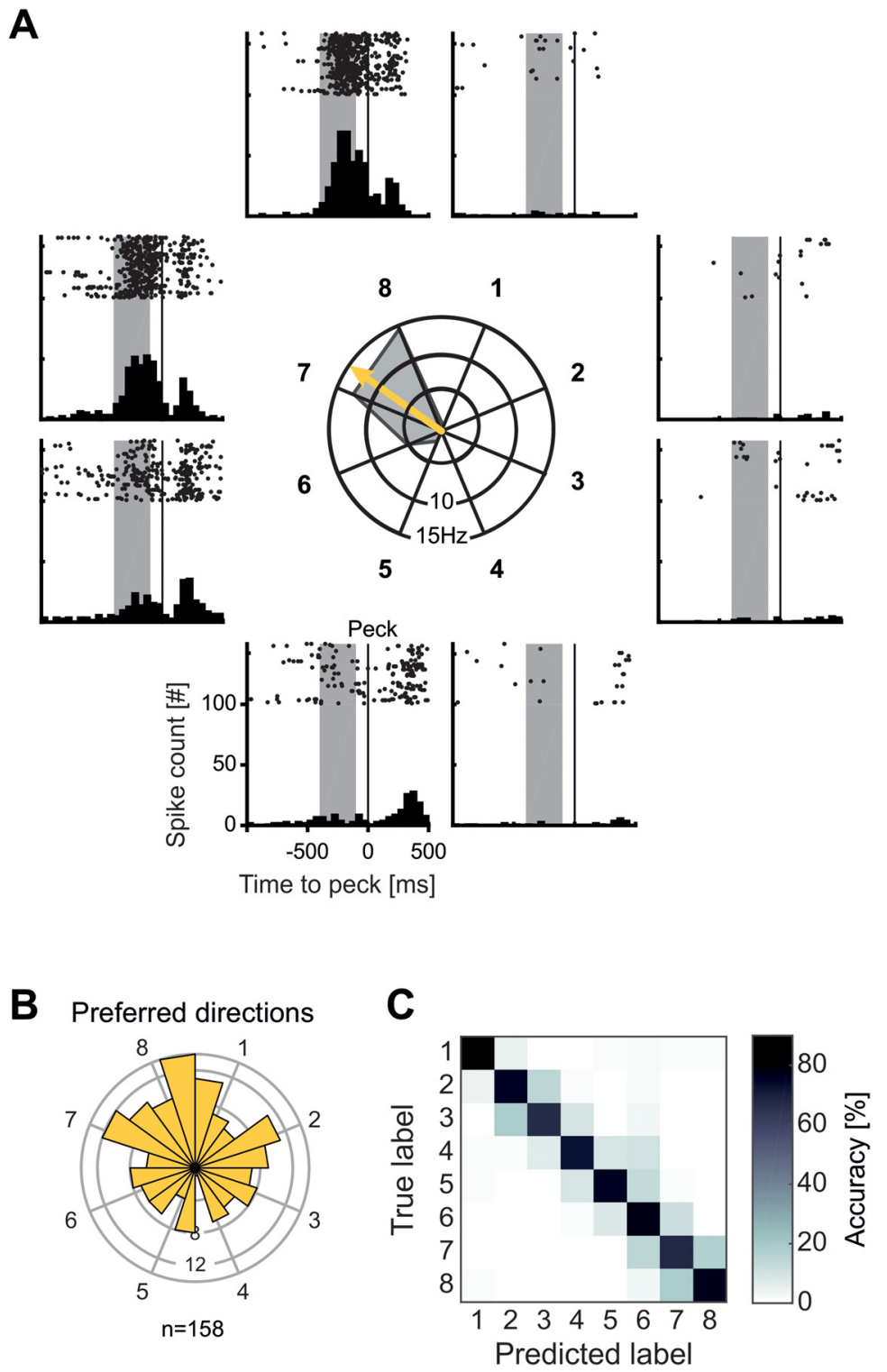

Figure 5. Neuronal responses to target locations during motion execution. $\boldsymbol{A}$, Example neuron with selective activity during movement to target locations. Central polar plot shows the average firing rate during movement to the eight target locations. Arrow indicates the preferred direction of the neuron. Around the polar plot each panel shows a dot-raster histogram with corresponding peristimulus time histogram (PSTH). In dot-raster histograms, each dot represents an action potential, and each line represents a trial. Each PSTH is the sum of action potentials in the dotraster histogram in bins of $50 \mathrm{~ms}$. Vertical lines represent the peck on the target location. Shaded area is the analysis window. The example neuron increases its activity selectively only for movement to target positions 7 and 8, with only minor increases to neighboring locations and no firing for all other positions. $\boldsymbol{B}$, Polar histogram of preferred directions of all execution-selective neurons $(n=158)$. Preferred directions were evenly distributed to various directions. C, Confusion matrix for SVM classifier accuracy during response execution. Each field represents for each true target label the respective percent of predictions of the classifier.

analysis shows that neurons exhibit the same kind of target side selectivity in both instructed and self-chosen target trials and are thus predictive of the crows' behavioral choice.

To explore choice-information of the entire population of neurons, we used a SVM classifier. If the selective activity in the planning period was related to the subsequent target side/location, activity between instructed and self-chosen target trials is expected to be similar, thus allowing an ideal observer to predict the choice in self-chosen target trials on basis of the neuronal activity. We trained an SVM classifier on the neuronal population activity of instructed trials and used the classifier to predict the self-chosen target side or location in the self-chosen condition. Figure $4 D$ shows the prediction performance for target side in self-chosen target trials. The classifier was able to predict the subsequently chosen target side significantly above chance level (i.e., average above the 95th percentile of permuted data). Moreover, the prediction of individual target locations in self-chosen target trials was also significantly higher than chance (Fig. 4E). This analysis shows that neuronal population activity during self-chosen target trials reliably predicted target side and location.

\section{Planning activity during error trials predicts actual target side}

To assess whether the side-selective activity in the planning period predicted the actually chosen side in error trials, we calculated an AUROC value for all side-selective neurons in the planning period $(n=84)$. We used both correct and error trials sorted according to the later response side. The average AUROC over the population of side-selective neurons during correct and error trials was significantly above 0.5 (mean AUROC: $0.62 ; p<0.05$ one-sided $t$ test). In a next step, we calculated the AUROC values using only error trials for all side-selective planningneurons that were recorded for at least five error trials per side $(n=17)$. The average AUROC value for the analyzed population of neurons was 0.55 and significantly higher that 0.5 $(p<0.05$ one-sided $t$ test). These results indicate that side-selective neurons during the planning period encode the planned response side regardless of the side instructed by the rule cues.

\section{Neurons encode target location during move- ment execution}

The activity of single neurons was modulated by target side and location not only during the planning phase, but also around the time of movement execution in the execution period. Figure $5 A$ shows the activity of an example neuron before and after a peck on the screen was registered. After offset of the rule cue (constituting the go signal), and before the crow's peck on target locations 7 and 8 , this neuron vigorously increases its firing rate. Except for a slight increase for pecks on target location 6 , there was virtually no increase in firing rate during this period for all other target locations. Hence, this neuron was only active during the execution of head movements toward specific target locations in the execution period.

To investigate selective activity during movement execution in the execution period on population level, we performed analyses of variance (four-factorial nested ANOVA with factors target side, target location, side cue type, and planning period length) while the crow was executing the planned movement. Analysis was performed on a 350-ms window ending $50 \mathrm{~ms}$ before a peck on the screen, accounting for the time when the bird was actively executing the movement and before the peck on the screen. Neurons with a significant factor for side cue type or planning 
period length were not considered exclusively execution-selective neurons because these neurons were encoding visual side cue features or exhibited timing related activity. Nearly $40 \%$ of all recorded neurons $(38.7 \%$ or $158 / 408)$, termed "execution-selective neurons," were modulated by the target location or the target side during movement execution in the execution period $(p<0.05$ for factors side and/or position, $p>0.05$ for factor side cue type and planning period length). The number of selective neurons were about evenly distributed between neurons selective for only the factor target side (49/158 "side-selective neurons"), only the factor target location (53/158 "location-selective neurons"), or both target side and location (56/158 side-and-location selective neurons). During motor execution, 30/408 neurons were affected by visual features $(p<0.05$ for factor rule cue type), $36 / 408$ neurons were affected by the factor planning time ( $p<0.05$ for factor planning period length), and 174/408 neurons were not selective for any factor.

We next calculated the preferred direction for all executionselective neurons $(158 / 408)$ in the same way as for the planning period by deriving individual directional vectors. The preferred directions for all execution-selective neurons can be seen in Figure $5 B$. In contrast to the findings for planning-selective neurons, execution-selective neurons were evenly tuned to both sides, without hemispheric lateralization ( $73 / 158$ or $46 \%$ preferring target locations on the right side; binomial test against equal distribution $p>0.05$ ).

In line with the analysis in the planning period, we trained a SVM classifier to predict the target location based on the neuronal population activity during movement execution. The classifier was able to predict the movement target significantly above chance with an average accuracy of $>70 \%$ and therefore higher than the 95th percentile of decoding accuracies obtained with shuffled data (95th accuracy percentile of shuffled data were around 20\%).

We also calculated a confusion matrix for the classifier performance for the execution period (Fig. 5C). Here, we found a strong diagonal of high prediction proportions, that is in coherence with the high overall decoding performance. Focusing on the misclassifications, most errors occur to neighboring target locations, regardless of their side. This indicates a representation of precise individual target locations during the movement execution.

\section{Comparison of tuning during motor planning and motor} execution

A subpopulation of neurons was selective in both the planning and execution period $(n=50)$. To evaluate whether the encoded locations were correlated, we calculated a circular-circular correlation between the preferred directions of these neurons (Berens, 2009). Neurons with selective activity in planning and execution period showed a significant correlation of their preferred directions $\left(p<0.05, \rho_{\mathrm{cc}}=0.31\right.$; angular difference: mean $=39.1^{\circ}$, $\left.\operatorname{std}=73.4^{\circ}\right)$.

We noticed that these planning-selective and execution-selective neurons tended to be tuned more precisely to single target locations during movement execution. Figure $6 A$ shows one example neuron with its response profile during the planning period (left polar plot) and during movement execution in the execution period (right polar plot). While this example neuron shows selective activity for target locations around target location 1 during both periods, the tuning is distinctly more sharply during movement execution.

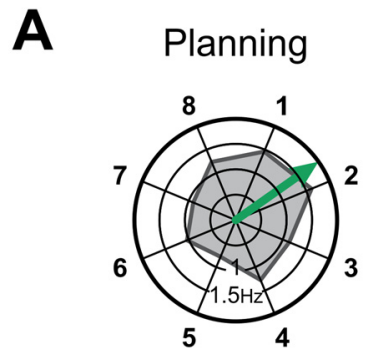

\section{Response}
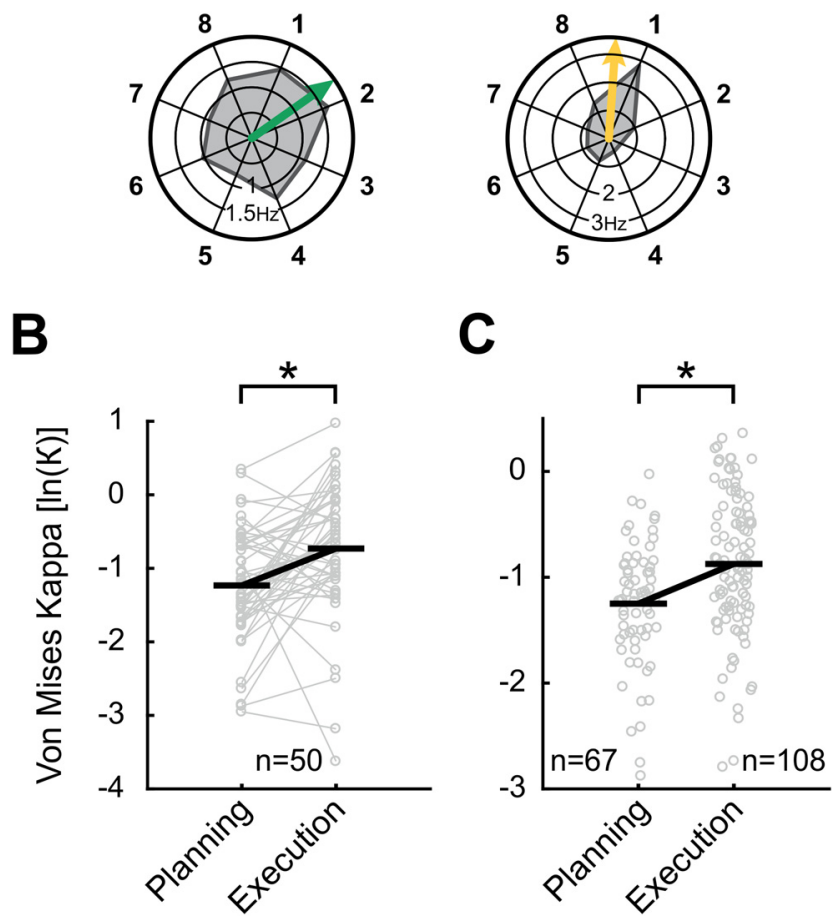

Figure 6. Comparison of tuning sharpness during planning and execution period. $\boldsymbol{A}$, Tuning of an example neuron selective during both planning and execution period. Polar plots show the average firing rate during planning and execution period for all eight target locations. Arrows represent preferred directions. While the neuron was selectively preferring target locations around location 1 both during planning and in the execution period, the tuning seems sharper during the execution period. $\boldsymbol{B}, \boldsymbol{C}$, Measure for tuning sharpness in planning and execution period for $(\boldsymbol{B})$ neurons selective in both periods and $(\boldsymbol{C})$ all remaining selective neurons. Horizontal black lines indicate average Kappa. For both neurons selective in $(\boldsymbol{B})$ both or $(\boldsymbol{C})$ only one period, tuning was significantly sharper during the execution period.

We wanted to analyze whether this effect of sharper tuning during movement execution was a general effect in our population of selective neurons. As a measure for the tuning sharpness of neurons, we calculated the Kappa $\kappa$ of a von Mises distribution for all neurons selective in the planning and/or execution period (Berens, 2009). A von Mises distribution is the circular equivalent of a Gaussian distribution, with Kappa $\kappa$ as an inverse equivalent for the SD $\sigma$. While large values of $\kappa$ indicate sharp tuning to distinct locations, a low $\kappa$ indicates broad tuning.

First, we compared the neurons selective in both planning and execution period (Fig. $6 B$ ) and found that $\kappa$ was significantly larger during movement executions $(n=50$; Wilcoxon signed-rank test $p<0.05$ ). Also, in the remaining population of neurons selective during either planning or execution period, $\kappa$ was significantly larger in the population of neurons selective in the execution period ( $n=67$ for planning and $n=108$ for execution period; MannWhitney $U$ test $p<0.05$; Fig. 6C). These analyses show that neurons that are selective during planning and execution and selective neurons in general are tuned more sharply during the execution period.

Time course of location preference throughout a trial To visualize the emergence of population activity of locationselective neurons over the time course of a trial, we calculated the sliding average firing rates (relative to baseline) to the 
A

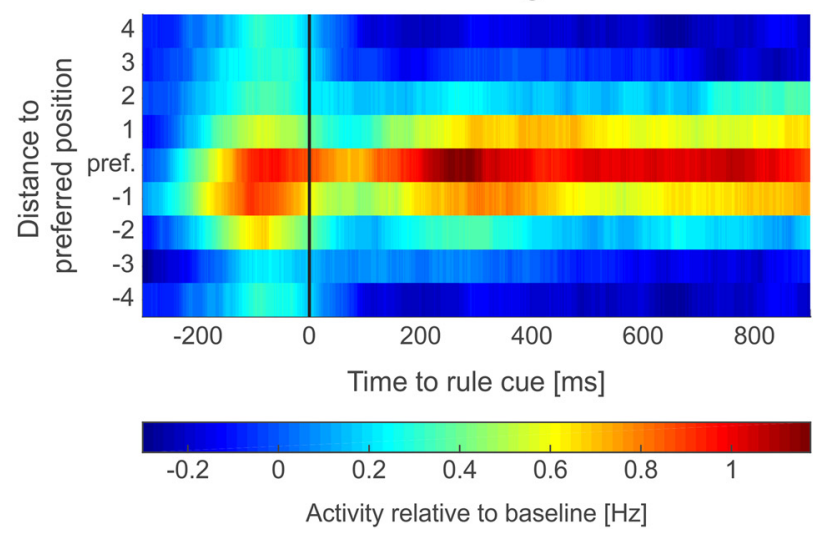

B

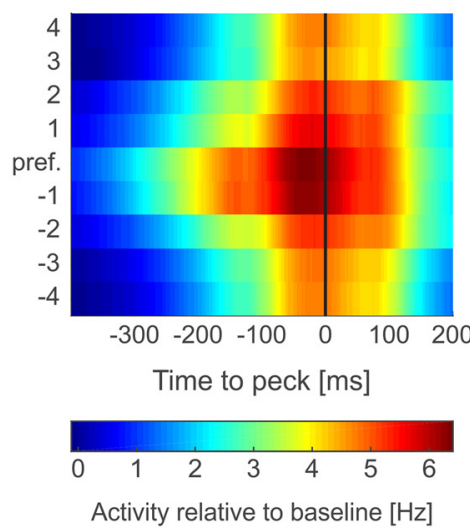

Figure 7. Emergence of target selectivity throughout a trial. Population firing rate relative to baseline of location-selective neurons $(n=142)$ throughout a trial. Across all neurons, neuronal activity (200-ms sliding window, 1-ms step size) was averaged across trials that instructed the preferred location of a respective neuron (center line) and the four clockwise and counterclockwise neighboring locations with increasing distance from the preferred location. $A$, Population activity during target cueing (negative time values) and subsequent motor planning. Data aligned to side cue onset $(0 \mathrm{~ms})$. $\boldsymbol{B}$, Population activity during movement execution. Data aligned to peck on the screen $(0 \mathrm{~ms})$.

preferred and neighboring non-preferred locations of all location-selective neurons (Cisek and Kalaska, 2005; Fig. 7). At the beginning of a trial when the two potential targets were displayed during the target cueing period (Fig. $7 A$, negative time values), an increase in activity to all target locations was seen, but with an emphasis on the subsequent preferred location during planning. Once the side cue was shown (at $0 \mathrm{~ms}$ in Fig. $7 A$ ), the firing rate increased in trials in which the preferred and, to a lesser extent, next-to preferred locations were instructed. In contrast, firing rate was suppressed below baseline in trials in which the preferred and instructed location were opposed. This pattern of activation was maintained throughout the analyzed period of the planning phase. Once the crow was prompted to execute a headbeak movement to the chosen target (Fig. $7 B$ ), an overall increase in discharges to all locations is seen. Nevertheless, discharges increased earlier and stronger if the motor target location and the preferred location coincided. Overall, an increase of spatial selectivity specifically during motor planning in comparison to the mere presentation of potential targets at trial start, and the execution of a movement toward a location at trial end was seen (Fig. 7).

\section{Discussion}

In the present study, we provide evidence for motor planning and motor execution activity in neurons of the corvid NCL. Neuronal activity in the planning period was behaviorally relevant and predicted the crows' upcoming choices. This neuronal code experienced a specification and sharpening during the transition from the planning to the execution periods. Below, we discuss the neuronal representations of head-beak actions as they progress from the planning to the execution stage.

\section{NCL activity during planning encodes the motor plan}

In the planning period, more than a fourth of the recorded NCL neurons prospectively encoded the future target side and/or one of the eight possible target locations. We ensured that these neurons were abstractly tuned to intended movement directions by excluding all neurons that showed visual selectivity to different types of side cues. We also excluded potential working memory activity by continuously presenting the two potential target locations and the side cue during the planning period: there was no need for the crows to memorize task factors throughout a trial. At the same time, the protocol forced the crows to prepare the head-beak movement right from the start of the planning period: the variable duration of the planning period in combination with a short response time window required crows to maintain gaze to the side cue in the center of the screen. Only by focusing on the central side cue, the crows were able to execute the movement as swiftly as possible once it was turned off as a go signal.

About half of the planning-selective neurons were categorically responsive to either the left or right side on which the subsequently correct target was located. At the population level, this allowed a classifier to predict the side of the thereafter chosen targets with high accuracy. Importantly, single neurons reliably predicted the prospective movement when the crows were confronted with neutral side cues during self-chosen target trials in which the crows needed to choose a target location. In this situation, the discharges of the population of selective neurons allowed a binary ROC classifier to predict the prospective target side, and an SVM classifier to correctly predict individual future target side and locations. The behavioral relevance of side-selective neurons was additionally confirmed by an analysis of error trials, it showed that the neurons encoded the later chosen target side also during error trials.

Given that NCL neurons encode behavioral rules (Veit and Nieder, 2013), we cannot exclude that such categorical left-versus-right planning activity may partly be because of a coding of the side rule. However, rule-selectivity cannot account for the response pattern of the other half of the selective neurons that were more specifically tuned to individual prospective target locations within a side. In addition, we found the majority of neurons preferring target locations contralateral to the recorded hemisphere, which is not to reconcile with a balanced representation of two equally important side rules. We therefore conclude that the responses of selective neurons during the planning period encoded the impending head-beak movement of the crows.

Motor planning activity has previously been suggested in the NCL of pigeons. When pigeons were trained in a visual categorization task to prepare movements to a left or a right pecking key in response to the grayscale value of a sample stimulus, NCL neuron activity was related to the subsequently chosen key side (Lengersdorf et al., 2014a). The authors interpreted this as neurons conveying a motor plan to the left or right (Lengersdorf et 
al., 2014a). However, alternative explanations related to sensory information cannot be ruled out. Since the pigeons' task was to always respond to the right choice key for dark stimuli, and to respond to the left choice key for bright stimuli, the observed activity might rather reflect the sample brightness or luminance categories. This potential confound was avoided in our study with crows by applying two sets of side cues that differed in appearance (color vs orientation) and excluding neurons responsive to cue appearance. Moreover, the involvement of NCL in the motor domain was also suggested by lesioning studies in pigeons. Several studies found an impairment in the execution of learned behavior after inactivation of NCL (Helduser and Güntürkün, 2012; Helduser et al., 2013; Lengersdorf et al., 2014b). However, since inactivation of the NCL likely blocks a multitude of task functions necessary to perform conditioned tasks (such as sensory and working memory functions), it is difficult to relate the observed performance disruptions to specific (pre)motor functions.

The current results in crows extend the apparent functional similarity between the convergently evolved avian NCL and the primate PFC into the premotor domain (Divac et al., 1985; Güntürkün, 2005; Nieder, 2017). Neurons in the primate PFC are well known to encode planned movements. This has been reported for different effector organs. For instance, PFC neurons encode the directions and target locations of intended eye (Asaad et al., 1998; Hasegawa et al., 1998; Markowitz et al., 2015) or limb movements (Niki and Watanabe, 1976; Saito et al., 2005; Yamagata et al., 2012), as well as the preparation of volitional vocalizations (Hage and Nieder, 2015, 2016; Gavrilov et al., 2017).

\section{Encoding of action execution}

While the bird was executing the head-beak movement toward one of the eight possible target locations, nearly $40 \%$ of all recorded neurons were tuned to movement direction. This large proportion of selective neurons allowed a classifier to predict the target location of the movement with a performance of $>70 \%$. In contrast to the planning period, all possible movement directions were evenly represented without a bias for a specific side. A similar uniform distribution of neuronal response vectors was reported in crows during a visual search task (Veit et al., 2017a). Overall, the high tuning resolution of movement directions in single cells and the large proportion of selective neurons suggest an important role of NCL also in encoding of head-beak movement directions.

We detected an interesting representational transformation during the transition from the planning to the execution period. While neurons represented the instructed side and were broadly tuned to impending target locations, the spatial tuning preference remained unchanged during movement execution period but became more precise and narrow. Remarkably, this effect was also present in individual neurons that were movementselective in both planning and execution periods and maintained their overall directional tuning vector. In contrast, this argues for the activation of a neuronal mechanism that sharpens tuning in individual neurons with progression from the planning to the execution stage.

The observed neuronal activity during motor execution is again reminiscent of the function of the primate PFC. PFC neurons also encode movement direction around the time of execution. For example, in monkeys trained on a rotatory oculomotor-delayed response task, a quarter of the recorded PFC neurons encoded the saccade direction either immediately before or after the saccade (Takeda and Funahashi, 2002). Similar results of PFC neurons encoding the saccade direction around time of execution were also reported in other studies (Funahashi et al., 1991; Asaad et al., 1998; Hasegawa et al., 1998).

\section{Dynamical spatial selectivity changes with task phases}

Our results in crows show that the avian NCL is not only involved in representing high-level sensory and cognitive task components. NCL activity also helps to transform behaviorallyrelevant information into motor plans and motor execution.

The emergence of neuronal signals throughout a trial suggests that NCL neurons simultaneously encode multiple movement options as long as the crow was not yet committed to a specific planned movement (i.e., before the side cue) or the execution thereof (i.e., when the response is performed). When the side cue was shown, the corresponding directional signal to the preferred location increase whereas the signal in trials with other locations became suppressed. This finding is reminiscent of the activity of monkey premotor cortex neurons that show an equivalent response pattern (albeit in a slight different task design; Cisek and Kalaska, 2005). The complementary findings in the avian brain suggest that the options for a forthcoming movement (motor decision) are encoded as potential actions that become resolved via competition between them in higher associative vertebrate brain areas (Cisek and Kalaska, 2010).

The lack of a clear distinction between motor planning and the actual motor execution mirrors findings in premotor areas of primates (Alexander and Crutcher, 1990; Wise et al., 1997; Crammond and Kalaska, 2000; Hoshi and Tanji, 2007). Even more, we observed both planning and execution processes in the activity of individual neurons, whose representations change from choosing abstract side information to executing head-beakrelated movement parameters. Such a temporal switching of single neurons between decision-related processes in choice tasks and subsequent movement related activity has also been observed in premotor areas of monkeys (Shen and Alexander, 1997; Crammond and Kalaska, 2000; Wallis and Miller, 2003; Romo et al., 2004; Cisek and Kalaska, 2005; Hoshi and Tanji, 2007; Klaes et al., 2011).

Both in the bird and mammal telencephalon, neurons in motor-related areas encode information about a stimulus, cognitive factors associated with the task, and subsequently motor variables (Wallis and Miller, 2003; Vallentin et al., 2012; Veit and Nieder, 2013; Veit et al., 2017b). Such functional heterogeneity at the level of single neurons in the independently and anatomically-distinctly evolved nidopallium of birds and cerebral cortex of mammals, may indicate that a separate action module does not exist in the vertebrate endbrain; if it exists, then it seems to be closely entwined with perceptual and cognitive processes (Lebedev and Wise, 2002; Cisek and Kalaska, 2010).

\section{References}

Alexander GE, Crutcher MD (1990) Neural representations of the target (goal) of visually guided arm movements in three motor areas of the monkey. J Neurophysiol 64:164-178.

Asaad WF, Rainer G, Miller EK (1998) Neural activity in the primate prefrontal cortex during associative learning. Neuron 21:1399-1407.

Berens P (2009) CircStat : a MATLAB toolbox for circular statistics. J Stat Softw 31.

Bird CD, Emery NJ (2009) Insightful problem solving and creative tool modification by captive nontool-using rooks. Proc Natl Acad Sci USA 106:10370-10375.

Brecht KF, Hage SR, Gavrilov N, Nieder A (2019) Volitional control of vocalizations in corvid songbirds. PLoS Biol 17:e3000375. 
Briscoe SD, Ragsdale CW (2018) Homology, neocortex, and the evolution of developmental mechanisms. Science 362:190-193.

Chandrasekaran C, Peixoto D, Newsome WT, Shenoy KV (2017) Laminar differences in decision-related neural activity in dorsal premotor cortex. Nat Commun 8:614

Chang CC, Lin CJ (2011) LIBSVM: a library for support vector machines. ACM Trans Intell Syst Technol 2:1-27.

Churchland MM, Shenoy KV (2007) Delay of movement caused by disruption of cortical preparatory activity. J Neurophysiol 97:348-359.

Cisek P, Kalaska JF (2005) Neural correlates of reaching decisions in dorsal premotor cortex: specification of multiple direction choices and final selection of action. Neuron 45:801-814.

Cisek P, Kalaska JF (2010) Neural mechanisms for interacting with a world full of action choices. Annu Rev Neurosci 33:269-298.

Crammond DJ, Kalaska JF (2000) Prior information in motor and premotor cortex: activity during the delay period and effect on pre-movement activity. J Neurophysiol 84:986-1005.

Davies MNO, Green PR (1994) Perception and motor control in birds (Davies MNO, Green PR, eds). Berlin, Heidelberg: Springer Berlin Heidelberg.

Ditz HM, Nieder A (2015) Neurons selective to the number of visual items in the corvid songbird endbrain. Proc Natl Acad Sci USA 112:7827-7832.

Ditz HM, Nieder A (2016) Sensory and working memory representations of small and large numerosities in the crow endbrain. J Neurosci 36:1204412052.

Ditz HM, Nieder A (2020) Format-dependent and format-independent representation of sequential and simultaneous numerosity in the crow endbrain. Nat Commun 11:686.

Divac I, Mogensen J, Björklund A (1985) The prefrontal "cortex" in the pigeon. Biochemical evidence. Brain Res 332:365-368.

Dubbeldam JL (2000) Motor control system. In: Sturkie's avian physiology (Whittow G, ed), Ed 5, pp 83-99. Cambridge: Academic Press.

Dykes M, Klarer A, Porter B, Rose J, Colombo M (2018) Neurons in the pigeon nidopallium caudolaterale display value-related activity. Sci Rep 8:5377.

Elemans CPH (2014) The singer and the song: the neuromechanics of avian sound production. Curr Opin Neurobiol 28:172-178.

Funahashi S, Bruce CJ, Goldman-Rakic PS (1991) Neuronal activity related to saccadic eye movements in the monkey's dorsolateral prefrontal cortex. J Neurophysiol 65:1464-1483.

Gavrilov N, Hage SR, Nieder A (2017) Functional specialization of the primate frontal lobe during cognitive control of vocalizations. Cell Rep 21:2393-2406.

Gdowski MJ, Miller LE, Parrish T, Nenonene EK, Houk JC (2001) Context dependency in the globus pallidus internal segment during targeted arm movements. J Neurophysiol 85:998-1004.

Green DM, Swets JA (1966) Signal detection theory and psychophysics. New York: Wiley.

Güntürkün O (2005) The avian 'prefrontal cortex' and cognition. Curr Opin Neurobiol 15:686-693.

Hage SR, Nieder A (2015) Audio-vocal interaction in single neurons of the monkey ventrolateral prefrontal cortex. J Neurosci 35:7030-7040.

Hage SR, Nieder A (2016) Dual neural network model for the evolution of speech and language. Trends Neurosci 39:813-829.

Hasegawa R, Sawaguchi T, Kubota K (1998) Monkey prefrontal neuronal activity coding the forthcoming saccade in an oculomotor delayed matching-to-sample task. J Neurophysiol 79:322-333.

Helduser S, Güntürkün O (2012) Neural substrates for serial reaction time tasks in pigeons. Behav Brain Res 230:132-143.

Helduser S, Cheng S, Güntürkün O (2013) Identification of two forebrain structures that mediate execution of memorized sequences in the pigeon. J Neurophysiol 109:958-968.

Hoffmann A, Rüttler V, Nieder A (2011) Ontogeny of object permanence and object tracking in the carrion crow, Corvus corone. Anim Behav 82:359-367.

Hoshi E, Tanji J (2000) Integration of target and body-part information in the premotor cortex when planning action. Nature 408:466-470.

Hoshi E, Tanji J (2006) Differential involvement of neurons in the dorsal and ventral premotor cortex during processing of visual signals for action planning. J Neurophysiol 95:3596-3616.
Hoshi E, Tanji J (2007) Distinctions between dorsal and ventral premotor areas: anatomical connectivity and functional properties. Curr Opin Neurobiol 17:234-242.

Iwaniuk AN, Wylie DR (2020) Sensory systems in birds: what we have learned from studying sensory specialists. J Comp Neurol 528:29022918.

Kabadayi C, Taylor LA, von Bayern AMP, Osvath M (2016) Ravens, New Caledonian crows and jackdaws parallel great apes in motor self-regulation despite smaller brains. R Soc Open Sci 3:160104.

Karten HJ (2015) Vertebrate brains and evolutionary connectomics: on the origins of the mammalian 'neocortex'. Philos Trans R Soc Lond B Biol Sc 370:20150060

Kersten Y, Friedrich-Müller B, Nieder A (2021) A histological study of the song system of the carrion crow (Corvus corone). J Comp Neurol. doi: $10.1002 / \mathrm{cne} .25112$.

Klaes C, Westendorff S, Chakrabarti S, Gail A (2011) Choosing goals, not rules: deciding among rule-based action plans. Neuron 70:536-548.

Kröner S, Güntürkün O (1999) Afferent and efferent connections of the caudolateral neostriatum in the pigeon (Columba livia): a retro- and anterograde pathway tracing study. J Comp Neurol 407:228-260.

Lebedev MA, Wise SP (2002) Insights into seeing and grasping: distinguishing the neural correlates of perception and action. Behav Cogn Neurosci Rev 1:108-129.

Lee IH, Assad JA (2003) Putaminal activity for simple reactions or self-timed movements. J Neurophysiol 89:2528-2537.

Lengersdorf D, Pusch R, Güntürkün O, Stüttgen MC (2014a) Neurons in the pigeon nidopallium caudolaterale signal the selection and execution of perceptual decisions. Eur J Neurosci 40:3316-3312.

Lengersdorf D, Stüttgen MC, Uengoer M, Güntürkün O (2014b) Transient inactivation of the pigeon hippocampus or the nidopallium caudolaterale during extinction learning impairs extinction retrieval in an appetitive conditioning paradigm. Behav Brain Res 265:93-100.

Markowitz DA, Curtis CE, Pesaran B (2015) Multiple component networks support working memory in prefrontal cortex. Proc Natl Acad Sci USA 112:11084-11089.

McDonald JH (2014) Handbook of Biological Statistics (3rd ed.). Baltimore: Sparky House Publishing.

Miller R, Boeckle M, Jelbert SA, Frohnwieser A, Wascher CAF, Clayton NS (2019) Self-control in crows, parrots and nonhuman primates. Wiley Interdiscip Rev Cogn Sci 10:e1504.

Moll FW, Nieder A (2015) Cross-modal associative mnemonic signals in crow endbrain neurons. Curr Biol 25:2196-2201.

Murphy K, James LS, Sakata JT, Prather JF (2017) Advantages of comparative studies in songbirds to understand the neural basis of sensorimotor integration. J Neurophysiol 118:800-816.

Nakayama Y, Yamagata T, Tanji J, Hoshi E (2008) Transformation of a virtual action plan into a motor plan in the premotor cortex. J Neurosci 28:10287-10297.

Nieder A (2017) Inside the corvid brain-probing the physiology of cognition in crows. Curr Opin Behav Sci 16:8-14.

Nieder A, Wagener L, Rinnert P (2020) A neural correlate of sensory consciousness in a corvid bird. Science 369:1626-1629.

Niki H, Watanabe M (1976) Prefrontal unit activity and delayed response: relation to cue location versus direction of response. Brain Res 105:7988.

Rinnert P, Kirschhock ME, Nieder A (2019) Neuronal correlates of spatial working memory in the endbrain of crows. Curr Biol 29:2616-2624.e4.

Romo R, Hernández A, Zainos A (2004) Neuronal correlates of a perceptual decision in ventral premotor cortex. Neuron 41:165-173.

Russ BE, Orr LE, Cohen YE (2008) Prefrontal neurons predict choices during an auditory same-different task. Curr Biol 18:1483-1488.

Saito N, Mushiake H, Sakamoto K, Itoyama Y, Tanji J (2005) Representation of immediate and final behavioral goals in the monkey prefrontal cortex during an instructed delay period. Cereb Cortex 15:1535-1546.

Schmidt MF, Wild JM (2014) The respiratory-vocal system of songbirds: anatomy, physiology, and neural control. Prog Brain Res 212:297-335.

Sen S, Parishar P, Pundir AS, Reiner A, Iyengar S (2019) The expression of tyrosine hydroxylase and DARPP-32 in the house crow (Corvus splendens) brain. J Comp Neurol 527:1801-1836.

Shen L, Alexander GE (1997) Neural correlates of a spatial sensory-to-motor transformation in primary motor cortex. J Neurophysiol 77:1171-1194. 
Takeda K, Funahashi S (2002) Prefrontal task-related activity representing visual cue location or saccade direction in spatial working memory tasks. J Neurophysiol 87:567-588.

Thura D, Cisek P (2014) Deliberation and commitment in the premotor and primary motor cortex during dynamic decision making. Neuron 81:1401-1416.

Vallentin D, Bongard S, Nieder A (2012) Numerical rule coding in the prefrontal, premotor, and posterior parietal cortices of macaques. J Neurosci 32:6621-6630

Veit L, Nieder A (2013) Abstract rule neurons in the endbrain support intelligent behaviour in corvid songbirds. Nat Commun 4:2878.

Veit L, Hartmann K, Nieder A (2014) Neuronal correlates of visual working memory in the corvid endbrain. J Neurosci 34:7778-7786.

Veit L, Pidpruzhnykova G, Nieder A (2015) Associative learning rapidly establishes neuronal representations of upcoming behavioral choices in crows. Proc Natl Acad Sci USA 112:15208-15213.
Veit L, Hartmann K, Nieder A (2017a) Spatially tuned neurons in corvid nidopallium caudolaterale signal target position during visual search. Cereb Cortex 27:1103-1112.

Veit L, Pidpruzhnykova G, Nieder A (2017b) Learning recruits neurons representing previously established associations in the corvid endbrain. J Cogn Neurosci 29:1712-1724.

Wallis JD, Miller EK (2003) From rule to response: neuronal processes in the premotor and prefrontal cortex. J Neurophysiol 90:1790-1806.

Weir AAS, Chappell J, Kacelnik A (2002) Shaping of hooks in New Caledonian crows. Science 297:981.

Wise SP, Boussaoud D, Johnson PB, Caminiti R (1997) Premotor and parietal cortex: corticocortical connectivity and combinatorial computations. Annu Rev Neurosci 20:25-42.

Yamagata T, Nakayama Y, Tanji J, Hoshi E (2012) Distinct information representation and processing for goal-directed behavior in the dorsolateral and ventrolateral prefrontal cortex and the dorsal premotor cortex. J Neurosci 32:12934-12949. 